\title{
NUMERICAL MODELLING OF THE TRANSIENT \\ HEAT TRANSPORT IN 2D SILICON THIN FILM USING THE INTERVAL LATTICE BOLTZMANN METHOD
}

\author{
Alicja Piasecka Belkhayat, Anna Korczak \\ Institute of Computational Mechanics and Engineering \\ Silesian University of Technology, Poland \\ alicja.piasecka@polsl.pl,anna.korczak@polsl.pl
}

\begin{abstract}
In the paper the description of numerical analysis of the heat transfer process proceeding in a two-dimensional silicon thin film is presented. It is assumed that some parameters like relaxation time and boundary temperatures appearing in the mathematical model of the analyzed problem are given as intervals. The discussed problem has been solved using the interval form of the lattice Boltzmann method applying the rules of the directed interval arithmetics. In the final part of the paper, the results of numerical computations are shown.
\end{abstract}

Keywords: heat transport, the interval lattice Boltzmann method

\section{Boltzmann transport equation}

The Boltzmann transport equation (BTE) is one of the fundamental equations of solid state physics and takes the following form [1-3]

$$
\frac{\partial f}{\partial t}+\mathbf{v} \cdot \nabla f=\frac{f^{0}-f}{\tau_{r}}+g_{e f}
$$

where $f$ is the phonon distribution function, $f^{0}$ is the equilibrium distribution function given by the Bose-Einstein statistics, $\mathbf{v}$ is the phonon group velocity, $\tau_{r}$ is the relaxation time and $g_{e f}$ is the phonon generation rate due to electron-phonon scattering.

In order to take advantage of the simplifying assumption of the Debye model, the BTE can be transformed to an equation on carrier energy density of the following form $[1,4]$

$$
\frac{\partial e}{\partial t}+\mathbf{v} \cdot \nabla e=-\frac{e-e^{0}}{\tau_{r}}+q_{v}
$$

where $e$ is the phonon energy density, $e^{0}$ is the equilibrium phonon energy density and $q_{v}$ is the internal heat generation rate related to a unit of volume. 
The equation (2) must be supplemented by the boundary-initial conditions.

Using the Debye model the relation between phonon energy density and lattice temperature is given by the following formula

$$
e(T)=\left(\frac{9 \eta k_{b}}{\Theta_{D}^{3}} \int_{0}^{\Theta_{D} / T} \frac{z^{3}}{\exp (z)-1} \mathrm{~d} z\right) T^{4}
$$

where $\Theta_{D}$ is the Debye temperature of the solid, $k_{b}$ is the Boltzmann constant, $T$ is the lattice temperature while $\eta$ is the number density of oscillators and can be calculated using the formula

$$
\eta=\frac{1}{6 \pi^{2}}\left(\frac{k_{b} \Theta_{D}}{h \omega}\right)^{3}
$$

where $h$ is the Planck constant divided by $2 \pi$ and $\omega$ is the phonon frequency.

\section{Interval lattice Boltzmann method}

The interval lattice Boltzmann method (ILBM) is a discrete representation of the Boltzmann transport equation. For 2D problems the interval Boltzmann transport equation can be written as

$$
\frac{\partial \bar{e}}{\partial t}+\mathbf{v} \cdot \nabla \bar{e}=-\frac{\bar{e}-\bar{e}^{0}}{\bar{\tau}_{r}}+q_{v}
$$

where $\bar{e}$ is the interval phonon energy density, $\bar{e}^{0}$ is the interval equilibrium phonon energy density, $\bar{\tau}_{r}=\left[\tau_{r}^{-}, \tau_{r}^{+}\right]$is the interval relaxation time $[4,5]$.

Equation (5) must be supplemented by the boundary conditions, for example for a square domain which is considered in the paper

$$
\begin{cases}x=0,0 \leq y \leq L: & \bar{e}(0, y, t)=\bar{e}\left(\bar{T}_{b 1}\right) \\ x=L, 0 \leq y \leq L: & \bar{e}(L, y, t)=\bar{e}\left(\bar{T}_{b 2}\right) \\ y=0,0<x<L: & \bar{e}(x, 0, t)=\bar{e}\left(\bar{T}_{b 3}\right) \\ y=L, 0<x<L: & \bar{e}(x, L, t)=\bar{e}\left(\bar{T}_{b 4}\right)\end{cases}
$$

and the initial condition of the following form:

$$
t=0: \quad \bar{e}(x, y, 0)=\bar{e}\left(T_{0}\right)
$$

where $\bar{T}_{b 1}=\left[T_{b 1}^{-}, T_{b 1}^{+}\right], \bar{T}_{b 2}=\left[T_{b 2}^{-}, T_{b 2}^{+}\right], \bar{T}_{b 3}=\left[T_{b 3}^{-}, T_{b 3}^{+}\right]$and $\bar{T}_{b 4}=\left[T_{b 4}^{-}, T_{b 4}^{+}\right]$are the given interval boundary temperatures, $T_{0}$ is the initial temperature. 
For a two-dimensional 9-speed model the discrete phonon velocities are expressed as [1]

$$
\mathbf{c}_{d}=\left\{\begin{array}{cc}
(0,0), & d=0 \\
(\cos [(d-1) \pi / 2], \sin [(d-1) \pi / 2]), & d=1, \ldots, 4 \\
\sqrt{2}(\cos [(d-5) \pi / 2+\pi / 4], \sin [(d-5) \pi / 2+\pi / 4]) c, & d=5, \ldots, 8
\end{array}\right.
$$

where $c=\Delta x / \Delta t=\Delta y / \Delta t$ is the lattice speed, $\Delta x$ and $\Delta y$ are the lattice distances from site to site, $\Delta t=t^{f+1}-t^{f}$ is the time step needed for a phonon to travel from one lattice site to the neighboring lattice site and $d$ is the direction.

The interval lattice Boltzmann method algorithm has been used to solve the analyzed problem $[1,6,7]$. The ILBM discretizes the space domain considered by defining lattice sites where the phonon energy density is calculated.

The lattice is a network of discrete points arranged in a regular mesh with phonons located in lattice sites. Phonons can travel only to neighboring lattice sites by ballistically traveling with a certain velocity and collide with other phonons residing at these sites according to Figure $1[1]$.

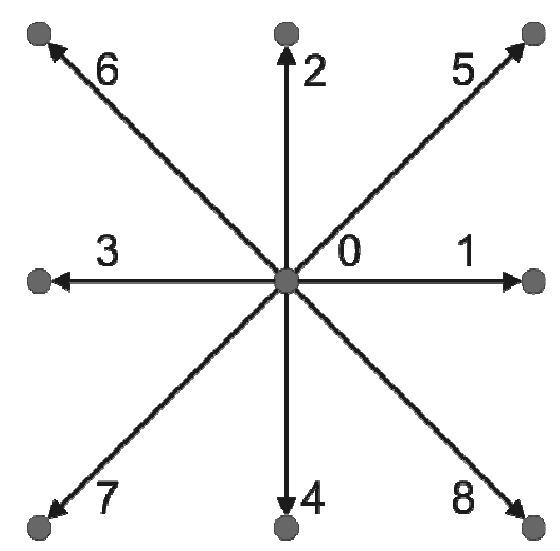

Fig. 1. Two-dimensional 9-speed (D2Q9) lattice Boltzmann model

The discrete set of propagation velocities in the main lattice directions can be defined as (see (8))

$$
\begin{array}{ll}
\mathbf{c}_{0}=(0,0) & \mathbf{c}_{1}=(c, 0) \\
\mathbf{c}_{2}=(0, c) & \mathbf{c}_{3}=(-c, 0) \\
\mathbf{c}_{4}=(0,-c) & \mathbf{c}_{5}=(c, c) \\
\mathbf{c}_{6}=(-c, c) & \mathbf{c}_{7}=(-c,-c) \\
\mathbf{c}_{8}=(c,-c) &
\end{array}
$$


In the interval lattice Boltzmann method is needed to solve nine equations allowing one to compute phonon energy in different lattice nodes according to the following equations:

$$
\begin{aligned}
& \frac{\partial \bar{e}_{0}}{\partial t}=-\frac{\bar{e}_{0}-\bar{e}_{0}^{0}}{\left[\tau_{r}^{-}, \tau_{r}^{+}\right]}+q_{v} \\
& \frac{\partial \bar{e}_{1}}{\partial t}+c \frac{\partial \bar{e}_{1}}{\partial x}=-\frac{\bar{e}_{1}-\bar{e}_{1}^{0}}{\left[\tau_{r}^{-}, \tau_{r}^{+}\right]}+q_{v} \\
& \frac{\partial \bar{e}_{2}}{\partial t}+c \frac{\partial \bar{e}_{2}}{\partial y}=-\frac{\bar{e}_{2}-\bar{e}_{2}^{0}}{\left[\tau_{r}^{-}, \tau_{r}^{+}\right]}+q_{v} \\
& \frac{\partial \bar{e}_{3}}{\partial t}-c \frac{\partial \bar{e}_{3}}{\partial x}=-\frac{\bar{e}_{3}-\bar{e}_{3}^{0}}{\left[\tau_{r}^{-}, \tau_{r}^{+}\right]}+q_{v} \\
& \frac{\partial \bar{e}_{4}}{\partial t}-c \frac{\partial \bar{e}_{4}}{\partial y}=-\frac{\bar{e}_{4}-\bar{e}_{4}^{0}}{\left[\tau_{r}^{-}, \tau_{r}^{+}\right]}+q_{v} \\
& \frac{\partial \bar{e}_{5}}{\partial t}+c \frac{\partial \bar{e}_{5}}{\partial x}+c \frac{\partial \bar{e}_{5}}{\partial y}=-\frac{\bar{e}_{5}-\bar{e}_{5}^{0}}{\left[\tau_{r}^{-}, \tau_{r}^{+}\right]}+q_{v} \\
& \frac{\partial \bar{e}_{6}}{\partial t}-c \frac{\partial \bar{e}_{6}}{\partial x}+c \frac{\partial \bar{e}_{6}}{\partial y}=-\frac{\bar{e}_{6}-\bar{e}_{6}^{0}}{\left[\tau_{r}^{-}, \tau_{r}^{+}\right]}+q_{v} \\
& \frac{\partial \bar{e}_{7}}{\partial t}-c \frac{\partial \bar{e}_{7}}{\partial x}-c \frac{\partial \bar{e}_{7}}{\partial y}=-\frac{\bar{e}_{7}-\bar{e}_{7}^{0}}{\left[\tau_{r}^{-}, \tau_{r}^{+}\right]}+q_{v} \\
& \frac{\partial \bar{e}_{8}}{\partial t}+c \frac{\partial \bar{e}_{8}}{\partial x}-c \frac{\partial \bar{e}_{8}}{\partial y}=-\frac{\bar{e}_{8}-\bar{e}_{8}^{0}}{\left[\tau_{r}^{-}, \tau_{r}^{+}\right]}+q_{v}
\end{aligned}
$$

The approximation of the first derivatives using right-hand and left-hand sides differential quotients is written as

$$
\frac{\partial \bar{e}_{d}}{\partial t}=\frac{\bar{e}_{d}(x, y, t+\Delta t)-\bar{e}_{d}(x, y, t)}{\Delta t} d=0,1, \ldots, 8
$$

and

$$
\begin{array}{ll}
\frac{\partial \bar{e}_{d}}{\partial x}=\frac{\bar{e}_{d}(x+\Delta x, y, t+\Delta t)-\bar{e}_{d}(x, y, t+\Delta t)}{\Delta x} & d=1,3,5, \ldots, 8 \\
\frac{\partial \bar{e}_{d}}{\partial y}=\frac{\bar{e}_{d}(x, y+\Delta y, t+\Delta t)-\bar{e}_{d}(x, y, t+\Delta t)}{\Delta y} & d=2,4,5, \ldots, 8
\end{array}
$$


Thus one obtains the approximate form of the interval Boltzmann transport equations for a two-dimensional problem in nine directions of the lattice $[1,2]$

$$
\left\{\begin{array}{l}
\left(\bar{e}_{0}\right)_{i, j}^{f+1}=\left(1-\Delta t / \bar{\tau}_{r}\right)\left(\bar{e}_{0}\right)_{i, j}^{f}+\Delta t / \bar{\tau}_{r} \cdot\left(\bar{e}_{0}^{0}\right)_{i, j}^{f}+\Delta t q_{v} \\
\left(\bar{e}_{1}\right)_{i+1, j}^{f+1}=\left(1-\Delta t / \bar{\tau}_{r}\right)\left(\bar{e}_{1}\right)_{i, j}^{f}+\Delta t / \bar{\tau}_{r} \cdot\left(\bar{e}_{1}^{0}\right)_{i, j}^{f}+\Delta t q_{v} \\
\left(\bar{e}_{2}\right)_{i, j+1}^{f+1}=\left(1-\Delta t / \bar{\tau}_{r}\right)\left(\bar{e}_{2}\right)_{i, j}^{f}+\Delta t / \bar{\tau}_{r} \cdot\left(\bar{e}_{2}^{0}\right)_{i, j}^{f}+\Delta t q_{v} \\
\left(\bar{e}_{3}\right)_{i-1, j}^{f+1}=\left(1-\Delta t / \bar{\tau}_{r}\right)\left(\bar{e}_{3}\right)_{i, j}^{f}+\Delta t / \bar{\tau}_{r} \cdot\left(\bar{e}_{3}^{0}\right)_{i, j}^{f}+\Delta t q_{v} \\
\left(\bar{e}_{4}\right)_{i, j-1}^{f+1}=\left(1-\Delta t / \bar{\tau}_{r}\right)\left(\bar{e}_{4}\right)_{i, j}^{f}+\Delta t / \bar{\tau}_{r} \cdot\left(\bar{e}_{4}^{0}\right)_{i, j}^{f}+\Delta t q_{v} \\
\left(\bar{e}_{5}\right)_{i+1, j+1}^{f+1}=\left(1-\Delta t / \bar{\tau}_{r}\right)\left(\bar{e}_{5}\right)_{i, j}^{f}+\Delta t / \bar{\tau}_{r} \cdot\left(\bar{e}_{5}^{0}\right)_{i, j}^{f}+\Delta t q_{v} \\
\left(\bar{e}_{6}\right)_{i-1, j+1}^{f+1}=\left(1-\Delta t / \bar{\tau}_{r}\right)\left(\bar{e}_{6}\right)_{i, j}^{f}+\Delta t / \bar{\tau}_{r} \cdot\left(\bar{e}_{6}^{0}\right)_{i, j}^{f}+\Delta t q_{v} \\
\left(\bar{e}_{7}\right)_{i-1, j-1}^{f+1}=\left(1-\Delta t / \bar{\tau}_{r}\right)\left(\bar{e}_{7}\right)_{i, j}^{f}+\Delta t / \bar{\tau}_{r} \cdot\left(\bar{e}_{7}^{0}\right)_{i, j}^{f}+\Delta t q_{v} \\
\left(\bar{e}_{8}\right)_{i+1, j-1}^{f+1}=\left(1-\Delta t / \bar{\tau}_{r}\right)\left(\bar{e}_{8}\right)_{i, j}^{f}+\Delta t / \bar{\tau}_{r} \cdot\left(\bar{e}_{8}^{0}\right)_{i, j}^{f}+\Delta t q_{v}
\end{array}\right.
$$

The total energy density is defined as the sum of discrete phonon energy densities in all the lattice directions

$$
\bar{e}_{i, j}^{f+1}=\sum_{d=0}^{8}\left(\bar{e}_{d}\right)_{i, j}^{f+1}
$$

The equilibrium phonon energy density is the same in all lattice directions and can be calculated using the formula

$$
\left(\bar{e}^{0}\right)_{i, j}^{f+1}=\frac{\bar{e}_{i, j}^{f+1}}{D}
$$

where $D$ is the number of all propagation directions in the lattice.

After subsequent computations the lattice temperature is determined using the following formula (see eq. (3))

$$
\bar{T}_{i, j}^{f+1}=\sqrt[4]{\bar{e}\left(\bar{T}_{i, j}^{f+1}\right) \Theta_{D}^{3} /\left(9 \eta k_{b} \int_{0}^{\Theta_{D} / \bar{T}_{i, j}^{f}} \frac{z^{3}}{\exp (z)-1} \mathrm{~d} z\right)}
$$




\section{Numerical examples}

As a numerical example the heat transport in a thin silicon film of the dimensions $200 \mathrm{~nm} \times 200 \mathrm{~nm}$ has been analyzed. The following input data have been introduced: the relaxation time $\bar{\tau}_{r}=[6.36675,6.69325] \mathrm{ps}$, the Debye temperature $\Theta_{D}=640 \mathrm{~K}$, the boundary conditions $\bar{T}_{b 1}=[780,820] \mathrm{K}$ and $\bar{T}_{b 2}=\bar{T}_{b 3}=\bar{T}_{b 4}=[292.5,307.5] \mathrm{K}$, the initial temperature $T_{0}=300 \mathrm{~K}$. The lattice step $\Delta x=\Delta y=20 \mathrm{~nm}$ and the time step $\Delta t=5 \mathrm{ps}$ have been assumed (see Fig. 2).

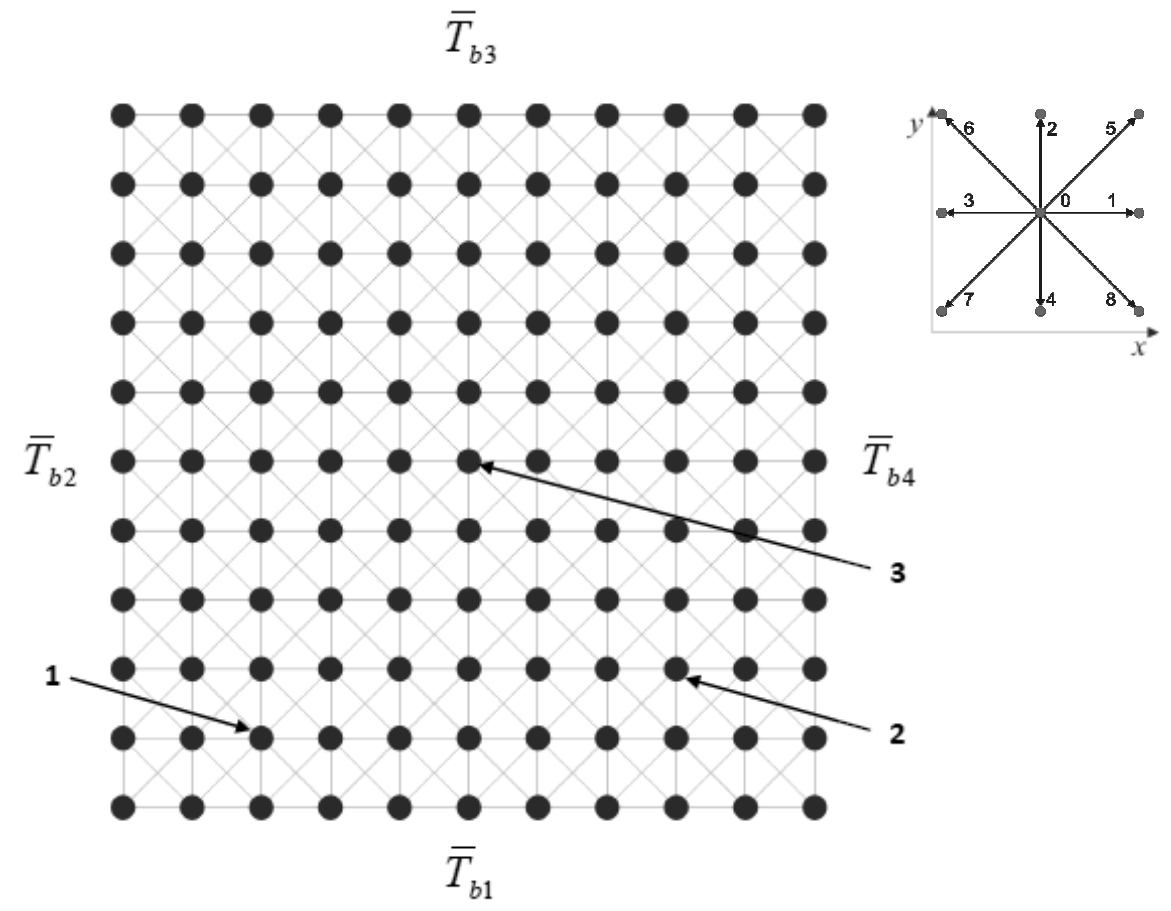

Fig. 2. Discretized domain

In the first example the internal heat generation rate related to a unit of volume $q_{v}=0$ has been assumed. Figure 3 presents the courses of the temperature function at the internal nodes $1(40 \mathrm{~nm}, 20 \mathrm{~nm}), 2(160 \mathrm{~nm}, 40 \mathrm{~nm})$ and $3(100 \mathrm{~nm}$, $100 \mathrm{~nm})$.

Figure 4 illustrates a comparison between the heating curves obtained using interval LBM and the results obtained using classical LBM for mean values of thermophysical parameters (dashed lines) at the same nodes as in Figure 3. 


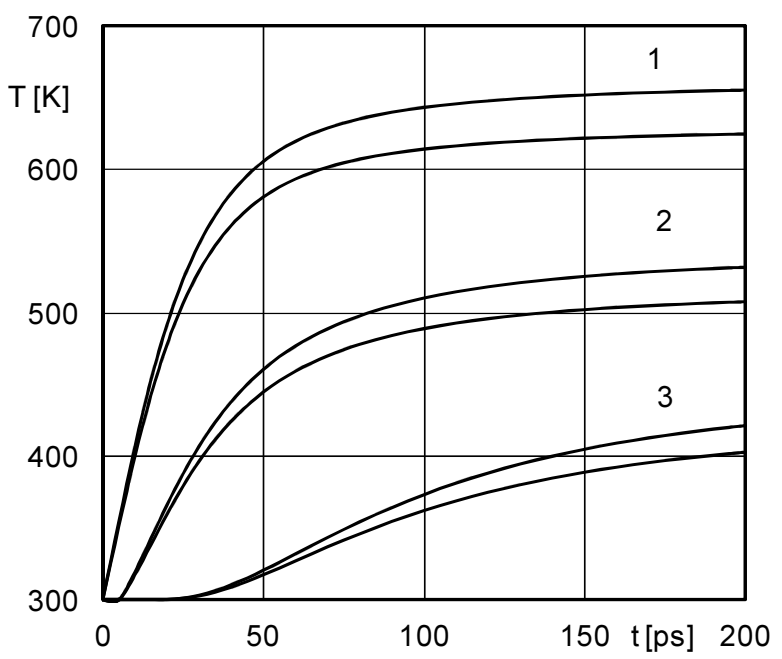

Fig. 3. Heating curves at internal nodes

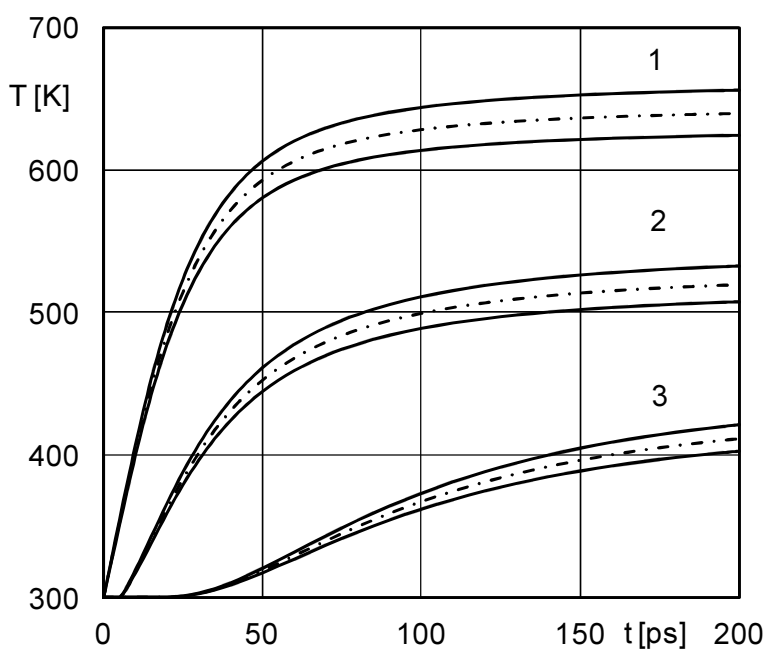

Fig. 4. Heating curves at internal nodes - comparison of interval LBM and classical LBM results

In the second example the internal heat generation rate related to a unit of volume has been assumed as $q_{v}=10^{18} \mathrm{~W} / \mathrm{m}^{3}$. Figure 5 shows the courses of the temperature function at the internal nodes. Figure 6 illustrates the interval heating curves at the same internal nodes for wider intervals of the boundary conditions $\left(\bar{T}_{b 1}=[760,840] \mathrm{K}, \bar{T}_{b 2}=\bar{T}_{b 3}=\bar{T}_{b 4}=[285,315] \mathrm{K}\right)$ and the relaxation time $\left(\bar{\tau}_{r}=[6.2035,6.8565] \mathrm{ps}\right)$. The interval temperatures are, of course, wider. 


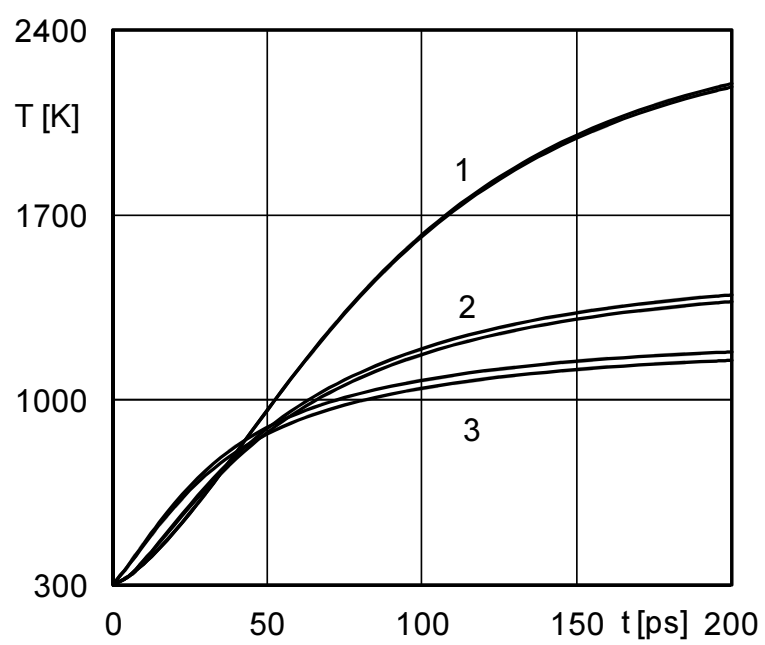

Fig. 5. The interval heating curves at internal nodes for $\mathrm{q}_{\mathrm{v}}=10^{18} \mathrm{~W} / \mathrm{m}^{3}$

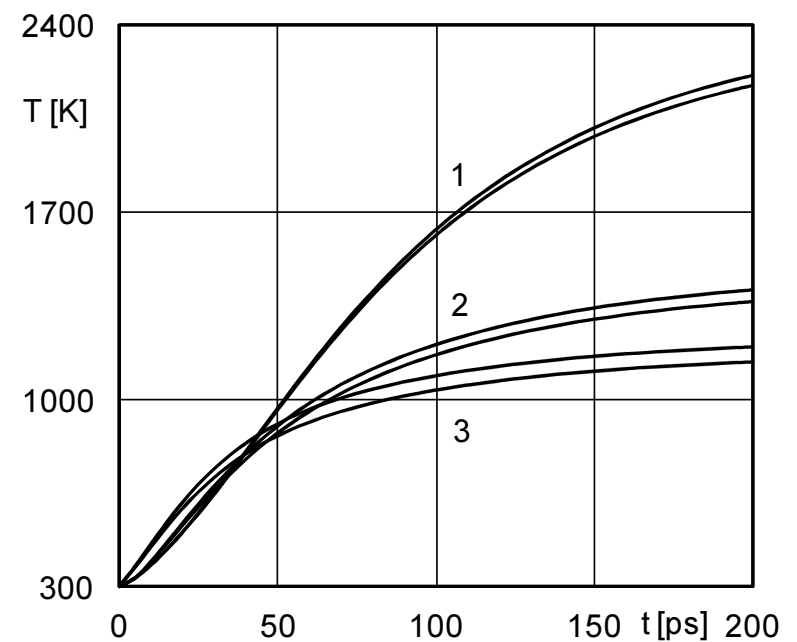

Fig. 6. The interval heating curves at internal nodes for wider intervals for $\mathrm{q}_{\mathrm{v}}=10^{18} \mathrm{~W} / \mathrm{m}^{3}$

\section{Conclusions}

In the paper the Boltzmann transport equation with the interval values of the relaxation time and the boundary conditions has been considered. The interval version of the lattice Boltzmann method for solving 2D problems has been presented. The generalization of LBM allows one to find the numerical solution in the 
interval form and such information may be important especially for the parameters which are estimated experimentally, for example the relaxation time.

The solutions obtained for mean values of the relaxation time and the boundary temperatures using the classical lattice Boltzmann method are always in intervals that are solutions of the interval lattice Boltzmann method. Increasing of the interval width of the relaxation time and boundary conditions widens temperature ranges.

\section{References}

[1] Escobar R.A., Ghai S.S., Jhon M.S., Amon C.H., Multi-length and time scale thermal transport using the lattice Boltzmann method with application to electronics cooling, Journal of Heat and Mass Transfer 2006, 49, 97-107.

[2] Joshi A.A., Majumdar A., Transient ballistic and diffusive phonon heat transport in thin films, Journal of Applied Physics 1993, 74(1), 31-39.

[3] Eshraghi M., Felicelli S.D., An implicit lattice Boltzmann model for heat conduction with phase change, International Journal of Heat and Mass Transfer 2012, 55, 2420-2428.

[4] Markov S.M., On directed interval arithmetic and its applications, Journal of Universal Computer Science 1995, 1, 514-526.

[5] Neumaier A., Interval Methods for System of Equations, Cambridge University Press, Cambridge, New York, Port Chester, Melbourne, Sydney 1990.

[6] Piasecka Belkhayat A., The interval lattice Boltzmann method for transient heat transport, Scientific Research of the Institute of Mathematics and Computer Science 2009, 1(8), 155-160.

[7] Piasecka Belkhayat A., Korczak A., Modelling of transient heat transport in one-dimensional crystalline solids using the interval lattice Boltzmann method, [in:] Recent Advances in Computational Mechanics, eds. T. Lodygowski, J. Rakowski, P. Litewka, Taylor \& Francis Group, A Balkema Book, London 2014, 363-368.

[8] Narumanchi S., Murthy J.Y., Amon C.H., Simulation of unsteady small heat source effects in submicron heat conduction, Journal of Heat Transfer 2003, 123, 896-903. 